\title{
Pain Research: Can Paradigmatic Expansion Bridge The Demands of Medicine, Scientific Philosophy and Ethics?
}

James Giordano, Ph.D.

As we approach the $60^{\text {th }}$ anniversary of the formalized use of the randomized controlled trial (RCT), we recognize its numerous benefits to the study of pain. By design, the RCT controls for supposition of bias, which is important when attempting to objectify the highly subjective nature of pain. Developed from the disease model to evaluate increasingly sophisticated pharmacologic effects, the RCT reflects the Zeitgeist of both the scientific epistemology and medical culture of the time. In many ways, it remains a methodologic standard, particularly when examining focal, specific effects, within populations in which sample randomization is appropriate (1). However, the RCT, while rigorous, has limitations that may warrant re-evaluation of its universal utility (2), necessitate co-implementation of supplemental research methodologies, and acknowledge an expanding conceptualization of pain using both disease-and illness-based models.

Pain is not a singular variable, but represents a condition resulting from somatosensory, cognitive and emotional events. Its subjective nature is difficult and often refractory to objective assessment and interpretation, and it phenomenologically changes as a consequence of time. Thus, durable chronic pain is physiologically, anatomically and subjectively distinct from acute/subacute pain. Chronic pain may persist as a result of continued chronic pathology or may progress due to plastic changes at numerous levels of the neuraxis, even after the apparent resolution of peripheral organic insult (3). Sev-

From Texas Medical Center, University of Texas, Houston, Texas and Moody Health Center, Texas Chiropractic College, Pasadena, Texas

Address Correspondence: James Giordano, Ph.D., Moody Health Center, Texas Chiropractic College, Pasadena, TX 77505 eral convergent disciplines have suggested that such neural changes, and their resultant constellation of cognitive and behavioral effects, can be influenced by multifocal variables across a hierarchy of interacting external and internal environments (4). Thus, chronic pain exists along a continuum that ranges from a manifestation of an underlying disease to an illness phenomenon that is very often defined by, and reciprocal to diverse patientspecific variables (Fig. 1). These are frequently bio-psychosocial and can influence the occurrence, severity and expression (i.e., subjective experience) of chronic pain, as well as become critical domains in which pain exerts effect(s) relevant to the lives of individual patients (5). An important step is to more clearly define these variables and include their assessment either as viable arms in RCTs, or as supplemental studies.

An understanding of the pain system illustrates the relatedness of sensory, perceptual and cognitive domains subserved by the anatomical substrates of chronic pain. Sensitization within this neuraxis can distort the relation of the pain phenomenon to the apparency of a peripheral lesion, making diagnosis, and therefore clinical therapeutic intervention, increasingly difficult. Furthermore, central sensitization can change activity at multiple neural substrates to create an emergent consciousness in which the patients' experience of both internal and external states is altered; thus, the patient with pain becomes the person defined by pain.

The goal of pain medicine is to decrease such effects on as many levels as possible, from the quantitative (e.g., sensory, perceptual, functional) to more abstractly qualitative (e.g., salutogenic, noetic). As shown in Fig. 2, it may be that while the RCT has effectively yielded information that is critical to the development of pharmacologic analgesics and implementation of pharmacologic protocols, a contemporary understanding of the relationship of brain-mind and mind-body to broader variable realms (i.e., cognition, culture) necessitates concomitant examination of other "levels" of data. Studying such complex, diversified substrates and their effects upon pain-related outcomes may be beyond the capacity of the RCT alone and may call for mixed-methods' approaches (2). The RCT may well remain the foundation upon which other studies can be built or interwoven. Within this proposed "crucible of research", evidence can be gained from correlational, qualitative and translational studies, as well. Of particular interest is the relationship between translational research and the RCT. The goal of translational research is to forge a bi-directional continuum between bench and bedside in which outcomes from one domain influence the direction of research in the other (6). Translational research may accumulate multiple lines of evidence (from the RCT through the qualitative) for application in clinical evaluation and/or therapy. Outcomes gained at the bedside should influence the direction of research both within RCTs as well as other types of studies. Such an approach utilizes both "bottomup" and "top-down" lines of inquiry and informational flow to evaluate processand patient-centered effects and diseaseand illness-based phenomenon. This approach is not heretical. In fact, quite to the contrary, it is simply that the growing body of knowledge about the complexity of the CNS and diversity of pain mechanisms implores an examination of evidentiary outcomes that are more extensive.

In both RCT and non-RCT approaches, there is issue regarding the ethical, legal and practical impact/effect(s) of "placebo" control and its comparative 


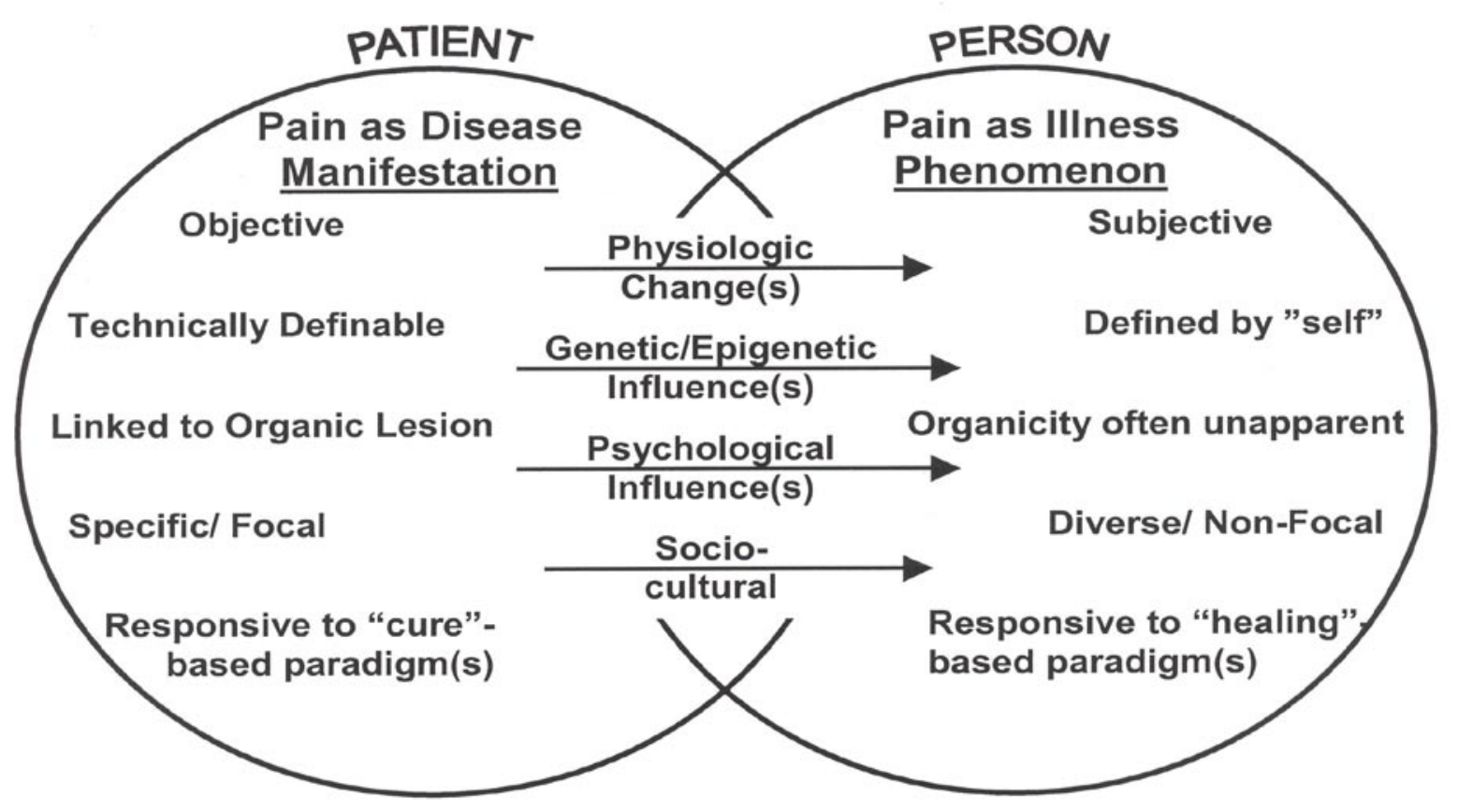

TIME

Fig. 1. Interactive spheres of effect and influence conceptualizing pain as a disease process versus pain as illness phenomenon. The disease is focal producing pain as a symptom in a patient in whom the pathologic process is definable by technical means that are at the disposal of the physician. Characteristically it responds to cure-based paradigms in which reduction/eradication of the underlying pathologic state concomitantly reduces/terminates the pain. If the disease cannot be cured or if the variety of depicted influences intervene and persist over time, the pain assumes phenomenological characteristics of a discrete illness that are diverse, and definable only by subjective evaluation of their effects upon the self. This may be responsive to healing-or palliative- based paradigms that seek to produce salutogenic or symptom-focal effects. The domains of disease and illness may be conjoint or may become relatively disjunctive as illness phenomena increase.

value (7). From an ethical standpoint, the concept of placebo implies deception or the intentional withholding of treatment. In part, this may be a semiotic issue in that placebo is not just inert or sham treatment but can be an agent that, under certain circumstances, may yield very powerful effects. Such "placebo effects" (or nocebo effects, if negative) are better considered as patient-centered, expectational, or meaning responses (8). Thus, a theoretical question arises when the outcomes of a study do not differ from placebo "control" effects: Did the placebo produce positive outcomes? If so, do such results truly reflect negative data? If held to methodological rigor and stringent analysis, might it not be possible that the therapeutic agency of a particular drug (and/or the circumstances of its provision and delivery) could be related, at least in part, to the ability to activate defined neural (and extra-neural) mechanisms with the apparent capacity to reduce both quantitative and qualitative symptoms related to pain? This topic is controversial, yet an accumulating body of evidence suggests that multiple cognitive variables (e.g., expectation, anticipation, belief) can influence the outcome(s) of blinded trials of pain therapies by engaging neuroanatomic substrates of pain modulation (9).
Can or should these findings be wholly disregarded when evaluating RCTs? This speaks to a wider set of philosophical and ethical issues.

Scarry (10) states that pain deconstructs lives. Thus, the patients' self (i.e., their personhood) is re-defined by the experience of pain and the needs that result. Such needs include the medical provision of agents and procedures that affect the pain experience, and respect for patients' autonomy obligates an understanding of their complexity and scope. Medicine has moved from a paternalistic to a more shared-experiential paradigm. The fiduciary relationship between physician and 


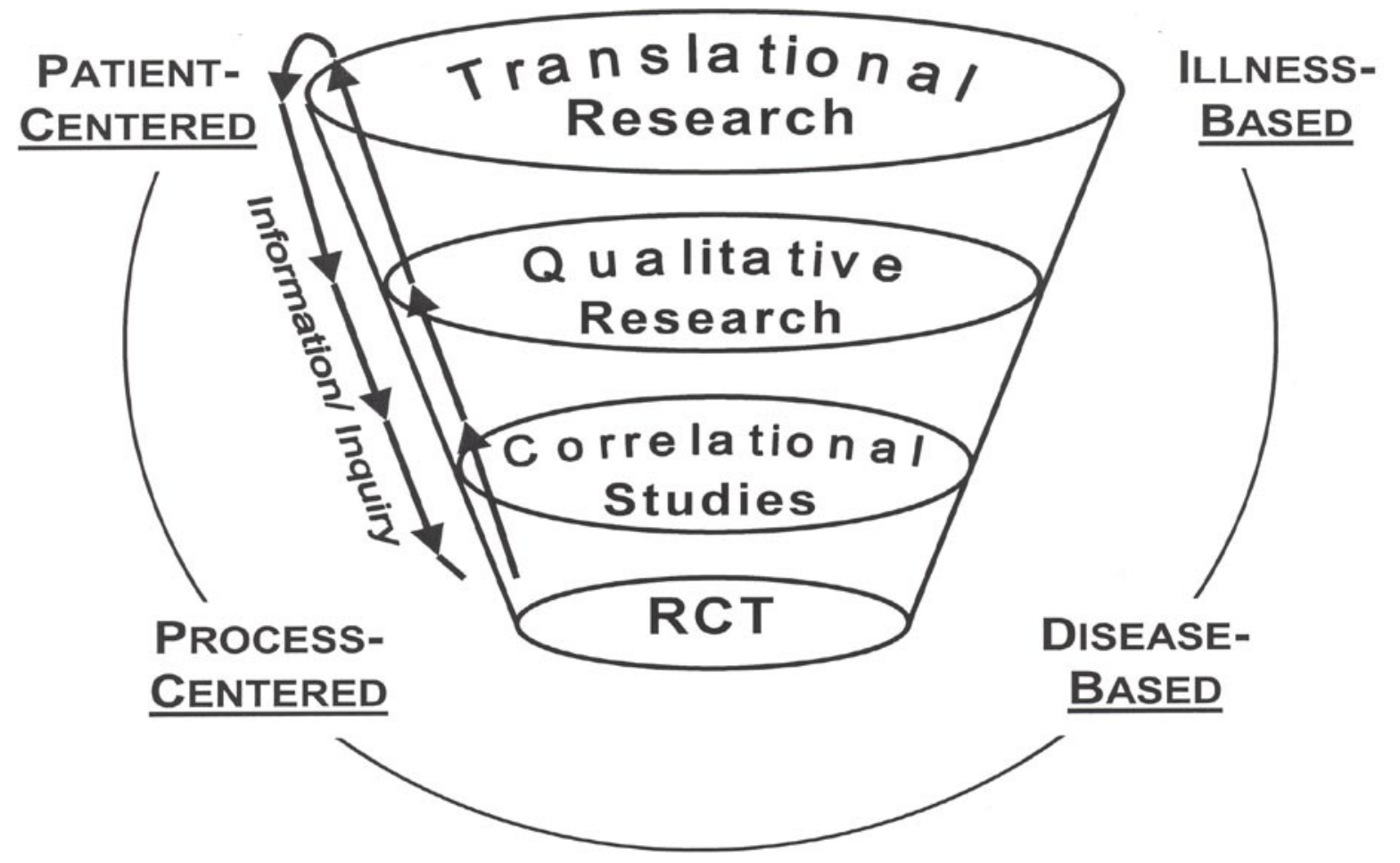

Fig. 2. The "crucible of research". The foundation of the crucible remains the RCT, which is well suited to evaluating process-centered and disease-based models of pain and pain therapy. Other types of studies may be structured upon the RCT (or used alternatively) to evaluate patient-centered and/or illness-based domains of pain. Lines of inquiry and informational flow are bi-directional and are dependent upon the domain of information studied and its relative orientation to other models of research. At each level, the acquisition of evidence must be gained through methods that are methodologically rigorous, but that may vary in their quantitative, qualitative or translational application.

patient mandates that physicians maintain the knowledge and competence to execute their professional role (11). Research provides this knowledge. Yet, such knowledge should not be limited to mechanistic aspects of treatment (although these are critical to evaluate the ratio of risk versus benefit) but should also include an understanding of "how" and in what domains the provision(s) of care is to occur. This affords patients access to the physician as both a source of "reasonable and necessary" information and as a guide in the access to venues for the appropriate paradigms of care. These paradigms may be curative and/or palliative, but their use must be evidence-based and derived from studies with rigorous and valid methodology (12). Equally important is that the lines of evidence be diverse so as to accommodate the proportional complexity of the variables involved in chronic pain and its treatment.

The research, scientific and clinical medical communities are inextricably bound to veracity as a cornerstone upon which other ethical duties (e.g., trust, virtue) and principles (e.g., respect for autonomy, beneficence, non-maleficence, justice) are structured (13). It is the imperative of science to seek truth, and the ethical obligation of the medical community to apply such truths justly, with respect for each patient so as to do no harm and facilitate over-arching good. Scientific philosophy should be free of dogmatism to remain committed to self-revision as a consequence of epistemic growth. Thus it is implicit that science and medicine must draw upon an ever-building epistemic capital to develop new ways of approaching enigmatic issues and solving problems.

Our current mechanistic understanding of brain, neurophilosophy of mind and recognition of chronic pain as a complex biocultural phenomenon compel an expansion and re-evaluation of research models such that no data are left unconsidered. 


\section{Author Affiliation \\ James Giordano, $\mathrm{PhD}$ \\ Visiting Fellow, Humanities in \\ Medicine Program \\ Texas Medical Center, University of \\ Texas \\ Houston, TX \\ Professor of Pathology \\ Director, Basic and Clinical Research \\ Moody Health Center \\ Texas Chiropractic College \\ Pasadena, TX 77505 \\ E-mail: Gsynapse22@aol.com}

\section{REFERENCES}

1. Meinert CL. Clinical Trials: Design, Conduct, and Analysis. Oxford University Press, New York, 1986.

2. Victora CG, Habicht JP, Bryce J. Evidence- based public health: Moving beyond randomized trials. Am J Public Health 2004; 94:400-405.

3. Cervero F, Laird JMA. From acute to chronic pain: Peripheral and central mechanisms. In Bountra C, Munglani R, Schmidt WK (eds.) Pain: Current Understanding, Emerging Therapies and Novel Approach es to Drug Discovery. Marcel Dekker Incorporated, New York, 2003.

4. Morris DB. The Culture of Pain. University of California Press, Berkeley, 1991.

5. Kleinman A, Brodwin PE, Good BJ et al. Pain as Human Experience: An Introduction. In DelVecchio-Good MJ, Brodwin PE, Good BJ et al (eds.) Pain as Human Experience: An Anthropological Perspective. University of California Press, Berkeley, 1992.

6. Marincola FM. Translational medicine: A two-way road. J Transl Med 2003; 1:1.

7. Kaptchuk TJ. Powerful placebo: The dark side of the randomized controlled trial. Lancet 1998; 351:1722-1725.

8. Moerman DE. The meaning response and the ethics of avoiding placebos. Eval Health Prof 2002; 25:399-409.

9. Fields HL, Price DD. Toward a neurobiology of placebo analgesia. In Harrington A (ed.) The Placebo Effect: An Interdisciplinary Exploration. Harvard University Press, Cambridge, 1997.

10. Scarry E. The Body in Pain. Oxford University Press, New York, 1985.

11. Rich BA. Physicians' legal duty to relieve suffering. West J Med 2001; 175:151-152.

12. Jonas WB. Evidence, ethics and the evaluation of global medicine. In Callahan D (ed.) The Role of Complementary and Alternative Medicine: Accommodating Pluralism. Georgetown University Press, Washington DC, 2002.

13. Bernat JL. Ethical Issues in Neurology. Butterworth-Heinemann, Boston, 2002. 